\title{
Enhancing Gas Permeability in Mixed Matrix Membranes through Tuning the Nanoparticle Properties
}

Shinji Kanehashi, ${ }^{1}$ George Q. Chen, ${ }^{1}$ Colin A. Scholes, ${ }^{1}$ Berkay Ozcelik, ${ }^{1}$ Carol Hua, ${ }^{2}$ Lachlan Ciddor, ${ }^{3}$ Peter D. Southon, ${ }^{2}$ Deanna M. D'Alessandro, ${ }^{2}$ and Sandra E. Kentish $^{1 *}$

1 Peter Cook Centre for Carbon Capture and Storage, Department of Chemical and Biomolecular Engineering, The University of Melbourne, Victoria 3010, Australia.

${ }^{2}$ School of Chemistry, The University of Sydney, New South Wales 2006, Australia.

${ }^{3}$ School of Chemistry, Monash University, Victoria 3800, Australia.

* Correspondence to Sandra E. Kentish

Tel : +61-3-8344-6682, Fax : +61-8344-4153

E-mail : sandraek@unimelb.edu.au 


\begin{abstract}
Mixed matrix membranes containing a variety of nanoparticles were fabricated by the solvent casting method using a commercial aromatic polyimide as the base polymer. All gas permeabilities increased with increasing particle loading with no reduction in the selectivity, reflecting adequate polymer/particle compatibility. Importantly, under such conditions the permeability enhancement depended only upon the pore volume within the particle, regardless of the particle chemistry or the morphology of the membrane structure. Remarkably, despite a range of membrane chemistries and filler loadings, this permeability enhancement could be readily described with a simple free volume relationship. The results suggest that nanoparticle porosity should be the focus of research into mixed matrix membrane structures. These results are likely to apply in all diffusivity dominated systems where the particle pore size is significantly larger than the penetrant size, as is the case with glassy polymers and many inorganic additives at low pressures.
\end{abstract}

Keywords: Mixed matrix membrane; Carbon dioxide; Polyimide; Gas separation; Free volume; 


\section{INTRODUCTION}

Mixed matrix membranes (MMMs) have been considered since the 1970s as an effective approach to improve the performance of both gas separation and water treatment polymeric membrane systems [1-3]. In this approach, nano-particulate fillers are added into the polymer phase, generally causing an enhancement in permeability and/or change in penetrant selectivity. A large number of MMMs using zeolites [4], silicas [5-7], carbons [8-10], metal-organic frameworks (MOFs) [11], zeolitic imidazolate frameworks (ZIFs) [12-14] and porous organic polymers (POPs) [15] have been shown to be promising candidates for gas separation applications. However, these MMMs are normally tested in isolation and rarely compared to each other. In the present work, a comparative study of gas separation using MMMs containing a variety of different nanoparticles has enabled important new insights into the factors that impact membrane performance.

The particles need to be well dispersed to guarantee the separation performance. One of the critical issues for MMMs is the presence of interfacial defects around the particles, which are caused by particle aggregation and poor particle-polymer interaction [16]. Such defects in the membrane can significantly reduce the gas selectivity and are also often related to increases in permeability. Therefore, MMM research has focused not only on the improvement of gas separation performance but also the preparation protocols and particle modifications to prevent or control these interfacial defects [17].

A number of workers have used computational approaches to evaluate the effect of nanoparticle properties [18, 19]. Their performance is also often predicted through mathematical models from the relatively simple Maxwell model [20] to others significantly more complex [21]. The Maxwell model [20] is considered appropriate for low particle concentrations (Equation 1); 


$$
P_{e f f}=P_{c}\left[\frac{P_{d}+2 P_{c}-2 \phi_{d}\left(P_{c}-P_{d}\right)}{P_{d}+2 P_{c}+\phi_{d}\left(P_{c}-P_{d}\right)}\right]
$$

where $P_{\text {eff }}$ is the effective permeability of the mixed matrix membrane, $\phi$ is the volume fraction and $P$ the permeability of the dispersed $(d)$ and continuous $(c)$ phases respectively. The Bruggeman [22] (Equation 2) and Lewis-Nielsen [23] (Equation 3) models were introduced to model systems with higher particle loadings:

$$
\begin{aligned}
& \frac{P_{e f f}}{P_{c}}=\frac{1}{\left(1-\phi_{d}\right)^{3}}\left[\frac{\left(P_{e f f} / P_{c}\right)-\alpha}{1-\alpha}\right]^{3} \\
& \frac{P_{e f f}}{P_{c}}=\frac{1+2 \phi_{d}[(\alpha-1) /(\alpha+2)]}{1-\phi_{d} \psi[(\alpha-1) /(\alpha+2)]}
\end{aligned}
$$

where $\alpha=\mathrm{P}_{\mathrm{d}} / \mathrm{P}_{\mathrm{c}}$ and $\psi=1+\left[\left(1-\phi_{\mathrm{m}}\right) / \phi_{\mathrm{m}}{ }^{2}\right] \phi_{\mathrm{d}}$, in which $\phi_{\mathrm{m}}$ is the maximum achievable volume fraction of the particles, which is affected by factors such as particle shape, size, size distribution, and agglomeration. Koros et al., also reported a modified Maxwell model that accounted for interfacial defects $(i)$ [16] (Equation 4):

$$
P=P_{c}\left[\frac{P_{e f f}+2 P_{c}-2\left(\phi_{d}+\phi_{i}\right)\left(P_{c}-P_{e f f}\right)}{P_{e f f}+2 P_{c}+\left(\phi_{d}+\phi_{i}\right)\left(P_{c}-P_{e f f}\right)}\right]
$$

However, an experimental evaluation of the impact of particle properties across a wide range of different nanoparticles has never been reported. In this work, we report for the first time the gas permeation properties of a series of MMMs in a glassy polymer substrate to determine the dominant particle properties and explain the permeation using a simple free volume-based model.

\section{EXPERIMENTAL}

\subsection{Materials}

The base polymer material used as a host matrix in this research is Matrimid ${ }^{\circledR} 5218$ (3,3,4,4-benzophenone tetracarboxylicdianhydride-diaminophenylidane) polyimide purchased 
from Huntsman Advanced Materials Americas Inc, America, in a powdered state. This polyimide was purified by solution and re-precipitation using methanol (Analytical reagent, Chem-Supply, Australia) and dichloromethane (DCM, Analytical reagent, Chem-Supply, Australia) to remove impurities. Carbon nanoparticles (Product Number; 699624 as Carbon A and 699632 as Carbon B), and zeolitic imidazolate framework $\left(\mathrm{ZIF}-8\left(\mathrm{Zn}(\mathrm{mim})_{2}, \mathrm{mim}=\right.\right.$ 2-methylimidazole), Basolite Z1200) as a filler phase were purchased from Aldrich Inc., America. Carbon $\mathrm{C}$ was produced from briquetted Victorian brown coal via mild steam activation at $800{ }^{\circ} \mathrm{C}$ for $90 \mathrm{~min}$, in a fixed bed reactor under a flow of $\mathrm{N}_{2} / \mathrm{H}_{2} \mathrm{O}(3 \mathrm{~L} / \mathrm{min}$ and $0.25 \mathrm{~cm}^{3} / \mathrm{min}$, respectively). $\mathrm{Cu}-\mathrm{BTC}\left(\left[\mathrm{Cu}_{3}(\mathrm{BTC})_{2}\right]_{3} \mathrm{H}_{2} \mathrm{O}\right.$, also known as HKUST-1 where BTC $=$ benzene-1,3,5-tricarboxylate) and a triarylamine-based POP (POP-2) were prepared according to the previous literature $[24,25]$. All materials were dried at $100^{\circ} \mathrm{C}$ under vacuum overnight to remove the moisture before use. The physical properties of the nanoparticles used in this study are summarized in Table 1.

\subsection{Mixed matrix membrane preparation}

MMMs were prepared by a solvent casting method. The loading of nanoparticles was varied from 0 to $30 \mathrm{wt} \%$ for carbon and metal organic framework MMMs and 0 to $20 \mathrm{wt} \%$ for POP-2 based on the weight of polymer. The nanoparticle loading was calculated based on the following equation;

$$
\text { Filler loading }(\mathrm{wt} \%)=\frac{w_{\text {filler }}}{w_{\text {polymer }}+w_{\text {filler }}} \times 100
$$

where $w_{\text {polymer }}$ and $w_{\text {filler }}$ are the weight of polymer and filler, respectively. Solutions of 3 $\mathrm{wt} \%$ filler and $3 \mathrm{wt} \%$ polymer in DCM were prepared separately by physical stirring using a magnetic stir bar overnight at room temperature, and each solution was then treated under ultrasonication (Unisonics, Australia) for 30 minutes within an iced water bath to maintain the temperature close to ambient. After ultrasonication, both homogeneous solutions were 
mixed together and cast directly onto a glass petri dish. This dish was then covered to prevent contamination and control the evaporation rate at room temperature for 24-48 hours. The membrane was then dried in a vacuum oven at $35^{\circ} \mathrm{C}$ for 24 hours and further dried at $100^{\circ} \mathrm{C}$ for 24 hours. Complete removal of the solvent was confirmed by thermogravimetric analysis (see Supporting Information). The thickness of the dried membranes varied from 60 to $100 \mu \mathrm{m}$. The MMMs became more rigid with increased filler loading, indicating that the nanoparticle increases the stiffness of the polyimide matrix. At loadings in excess of 30 wt\% filler, the membranes became too brittle to handle, and thus only mixed matrix membranes containing up to $30 \mathrm{wt} \%$ were tested for gas permeation.

\subsection{Characterization}

Nitrogen adsorption-desorption measurements of these nanoparticles were conducted on a ASAP2010 (Micromeritics, USA) apparatus at $77 \mathrm{~K}$. The internal surface area was then calculated by the BET method. The nanoparticle size was measured in an ethanol solution for the carbons and in water for the MOFs by dynamic light scattering (DLS) using a Zetasizer 2000 (Malvern, UK) instrument. The solution was sonicated for 10 minutes to break up agglomerates prior to this experiment.

The membrane density $(\rho)$ was determined based on the Archimedean principle at room temperature $\left(24 \pm 1^{\circ} \mathrm{C}\right)[26]$. Dense membranes were weighed using an analytical balance (Mettler Toledo AB204-5) in air (wair) and then in ethanol (Undenatured AR 100\%, Chem-Supply, Australia) $\left(w_{E t O H}\right)$. The membrane density of the base Matrimid was measured as $1.223 \pm 0.019 \mathrm{~g} / \mathrm{cm}^{3}$, which is in good agreement with the literature value of $1.225 \mathrm{~g} / \mathrm{cm}^{3}[27]$.

The volume fraction of carbon particle $\left(\phi_{\text {filler }}\right)$ in the membrane was calculated using the following equation: 


$$
\phi_{\text {filler }}=\frac{w_{\text {filler }}}{w_{\text {filler }}+\frac{\rho_{\text {filler }}}{\rho_{\text {polymer }}}\left(1-w_{\text {filler }}\right)}
$$

where $w_{\text {filler }}$ is the weight fraction of carbon particle added to the membrane. $\rho_{\text {polymer }}$ and $\rho_{\text {filler }}$ are the density of polymer and nanoparticle, respectively. This experiment was performed repeatedly using several different membranes to ensure reproducibility. As a comparison, the theoretical density ( $\rho_{\text {theory }}$ ) of the mixed matrix membranes was calculated using the following equation:

$$
\rho_{\text {theory }}=\frac{1}{\frac{1-w_{\text {filler }}}{\rho_{\text {polymer }}}+\frac{w_{\text {filler }}}{\rho_{\text {filler }}}}
$$

The difference between the theoretical and the measured density $\left(\rho_{\text {exp }}\right)$, provides an indication of the additional voids created by incompabitility between the nanoparticles and the surrounding polymer. This void volume fraction, or additional free volume, $\phi_{V}$ can be estimated from:

$$
\varphi_{V}=1-\left(\frac{\rho_{\mathrm{exp}}}{\rho_{\text {theo }}}\right)_{r y}=\left(\frac{\rho_{\text {he o } \bar{r}_{y} \rho_{\mathrm{e}}}}{\rho \text { the }}\right)_{\text {. }}
$$

The water uptake of membrane was determined by immersion in pure water at $35 \pm$ $1^{\circ} \mathrm{C}$ until the equiribrium water sorption was attained. The water uptake was calculated from:

$$
\text { Water uptake }=\frac{w_{w e t}-w_{d r y}}{w_{d r y}} \times 100
$$

where $w_{\text {wet }}$ and $w_{d r y}$ are the weight of a water-swollen membrane at equiribrium state and a well-dried.

The nanoparticle and membrane morphologies were imaged using a FEI Quanta FEG 200 scanning electron microscope (SEM, FEI Company, USA) operated at an accelerating voltage of $10 \mathrm{kV}$. The membrane sample was prepared by cleavage under liquid nitrogen to 
give a well-defined cross-section. All samples were dried and then sputtered with a thin layer of gold using a Dynavac Mini Sputter Coater (Dynavac, USA). The transmission electron microscope (TEM) images of nanoparticles were acquired using a FEI Tecnai TF20 instrument with an operating voltage of $200 \mathrm{kV}$.

\subsection{Pure gas permeation}

The pure gas permeability of $\mathrm{O}_{2}, \mathrm{~N}_{2}, \mathrm{CO}_{2}$, and $\mathrm{CH}_{4}$ were determined by the constant-volume/variable-pressure method, which measures the steady-state gas flux through a membrane of known thickness under a given pressure difference [28]. All permeation data were determined at $35^{\circ} \mathrm{C}$ and 2 atm using a minimum of two samples to ensure the reproducibility of the experimental results. The gas permeation measurement is performed with a leak rate of below $1.0 \times 10^{-6}$ torr/s, which is negligible in the case of well dried defect free samples. The gas permeation coefficient $P\left(\right.$ barrer $\left.=\times 10^{-10} \mathrm{~cm}^{3}(\mathrm{STP}) \mathrm{cm} /\left(\mathrm{cm}^{2} \cdot \mathrm{s} \cdot \mathrm{cmHg}\right)\right)$ was determined from the slope of the time-pressure curve at steady state. The ideal gas selectivity $(\alpha(\mathrm{A} / \mathrm{B}))$ of gas $\mathrm{A}$ over gas $\mathrm{B}$ was expressed as the ratio of their permeability coefficients $\left(P_{A}\right.$ and $\left.P_{B}\right)$.

$$
\alpha(A / B)=\frac{P_{A}}{P_{B}}
$$

\section{RESULTS AND DISCUSSION}

\subsection{Characterization}

The water uptake value of membranes prepared from $\mathrm{Cu}$-BTC was around 2.8 times higher than that of the base Matrimid, while that for a membrane prepared with POP-2 was $40 \%$ lower than that of Matrimid (Table 1). The data suggests the order of hydrophilicity of the MMMs to be $\mathrm{Cu}$-BTC > ZIF-8 > Carbon $\mathrm{C}>$ Carbon $\mathrm{B}>$ Matrimid > Carbon A > POP-2. 
The particle size distribution of the carbon (Carbon A, B, and C) and MOF (Cu-BTC and ZIF-8) nanoparticles are presented in Figure 1. We could not obtain a reasonable distribution for POP-2 due to persistent aggregation of the particles, so this data is not presented. However, SEM images indicated a particle size of less than $300 \mathrm{~nm}$ for this filler, consistent with the other fillers. A small particle size is desirable for composite membrane applications, as the selective layer is often less than $300 \mathrm{~nm}$ thick.

Figure 2 presents the cross-sectional scanning electron microscopy (SEM) images of $20 \mathrm{wt} \%$ MMMs. These pictures indicate that the fillers are well-dispersed in the polymer matrix but that the morphology changes significantly with the nature of the filler. Membranes prepared from more hydrophobic particles (as indicated by the water uptake) such as Carbon A and POP-2 appeared to show more compact structures relative to the more hydrophilic membranes. This is not unexpected, given that Matrimid itself is hydrophobic.

However, surprisingly, the voids between particles and polymer, calculated using Equation 8, in all cases remain below 5\% of the total volume (Figure 3). The void volume fraction increased with filler loading as might be expected (Figure 3a), but the very different hydrophobicity, and the resulting changes in SEM morphology, was not strongly correlated with this void volume fraction (Figure 3b). For example, POP-2 had the greatest apparent void volume, but this is not apparent from Figure 2.

The void volume fraction is calculated values based on the difference in the density between actual membrane and particle (Equation 8). These differences are small and hence any error is potentially magnified. These issues are aggravated by the fact that we use literature or manufacturer information for the particle density of Carbon A and B, ZIF-8 [13], and Cu-BTC [29-31]. Merkel et al. [32] also point out that the void volume fraction is difficult to 
estimate accurately using this approach. These large errors will contribute to the poor correlation between void volume and the SEM images. However, the fact that the void volume is consistently less than 5\% while some SEM images show very large voids of 100 $\mathrm{nm}$ in size, suggests that the morphological differences observed in the SEM images may be an artifact of the imaging process itself. It is likely that the true voids may be smaller than can be detected using SEM.

\subsection{Pure gas permeation}

Figure 4 presents the $\mathrm{CO}_{2} / \mathrm{CH}_{4}$ and $\mathrm{CO}_{2} / \mathrm{N}_{2}$ separation performance maps of MMMs using Matrimid as the base polymer together with other literature values (open circles). The $\mathrm{CO}_{2}$ permeability and $\mathrm{CO}_{2} / \mathrm{CH}_{4}$ selectivity of Matrimid are 7.3 Barrer and 35 respectively. The literature data for Matrimid-based MMMs is widely scattered. Some MMMs exhibit improvement in both selectivity and permeability, while others experience considerable selectivity losses. For example, Matrimid-based MMMs using mesoporous ZSM-5 zeolite show improved $\mathrm{CO}_{2} / \mathrm{CH}_{4}$ selectivity up to 67 [33]. Our MMMs also show slight improvement in the $\mathrm{CO}_{2} / \mathrm{CH}_{4}$ selectivities at low particle loadings, although most are within the range of experimental error. The selectivities of the MMMs using $10 \mathrm{wt} \%$ loading of Carbon B and Carbon $\mathrm{C}$ are 37 and 43 respectively, an increase of up to $25 \%$. An enhancement in the selectivity can relate to the molecular sieving properties of the fillers, or to rigidification of the polymer chains in the immediate vicinity of the nanoparticles[34]. The pore sizes of the nanoparticles used in the present work (Table 1) are much larger than the size of the penetrant molecules, so any increase in the present case would result from chain rigidification rather than molecular sieving. On the other hand, the slight decline in gas selectivity as the loading increases can result from the increase in void volume fraction described in Figure 3. Importantly, the fact that no major loss in selectivity is observed 
confirms that large particle-polymer interfacial defects are absent. This reinforces the conclusion that the large defects observed in the SEM images represent experimental artifacts. Conversely, the $\mathrm{CO}_{2}$ permeability of the MMMs enhances significantly with increasing filler loading, regardless of the identity of the nanoparticle.

Figure 5 presents the $\mathrm{CO}_{2}$ permeability enhancement of the MMMs as a function of the filler volume fraction (Equation 2) at $35^{\circ} \mathrm{C}$ and 2 atm. As evidenced from Figure 4, the $\mathrm{CO}_{2}$ permeability increases with filler loading. The enhancement for the MOF-incorporated MMMs at high volume fractions $(\phi>0.21)$ is significantly larger than that of the Carbon MMMs (i.e., Cu-BTC > ZIF-8 = POP-2 Carbon $\mathrm{C}>$ Carbon $\mathrm{B}>$ Carbon A). The permeability data was then analysed to determine which particle properties were most strongly aligned to the results. Particle surface area, porosity, size, mass fraction and hydrophobicity were considered in this analysis. Again, the strong correlation between the hydrophobicity of the particles and their morphology when embedded in the hydrophobic polymer (Figure 6a) was not reflected in the permeability data. Similarly, while other workers have shown a correlation between the mass fraction of a single nanoparticle and the permeability, we did not find a common relationship with the mass fraction when a range of nanoparticles was considered. Rather, we found that particle surface area, as determined by adsorption experiments provided the strongest correlation to the permeability enhancement (Figure 6b). This could suggest that surface diffusion, a common feature of permeation through inorganic materials $[35,36]$, may play a role. However, this surface area is in itself strongly correlated to the pore volume within an adsorbent material (Figure 7).

This led us to consider the very simple model developed originally by Fujita that relates penetrant diffusivity to the free volume within a system [37]. In systems that are diffusivity selective, which is true for both the base Matrimid and the fillers considered here, 
this simple model may be extended to relate permeability $(P)$ to the fractional free volume $(F F V)[38,39]$.

$$
P=A_{P} \exp \left(-\frac{B_{P}}{F F V}\right)
$$

where $A_{p}$ and $B_{p}$ are adjustable constants dependent upon both temperature and the nature of the penetrant type. The FFV of the Matrimid polymer can be characterized by the Bondi method [40];

$$
F F V=\frac{V-1.3 V_{W}}{V}
$$

where $V$ is the specific volume of polymer, and $V_{w}$ is the van der Waals volume. The calculated value for pure Matrimid was 0.167 , which is consistent with the literature [26, 41]. The fractional free volume of the filler was determined by multiplying the pore volume $\left(\mathrm{cm}^{3} / \mathrm{g}\right)$ as measured by the BET sorption analysis, by the particle density $\left(\mathrm{g} / \mathrm{cm}^{3}\right)$ [29].

The fractional free volume of the mixed matrix membrane is then simply determined from Equation (13):

$$
\text { Total } F F V=F F V_{\text {polymer }}\left(\phi_{\text {polymer }}\right)+F F V_{\text {filler }}\left(\phi_{\text {filler }}\right)
$$

Figure 8 presents the gas permeabilities for all membranes tested as a function of the inverse of the $F F V$. There is a remarkably strong correlation between permeability and $F F V$ on this basis, particularly given the wide range of nanoparticles used. These strong correlations are found regardless of the gas molecule. These results demonstrate that for membranes based on glassy polymers within this pressure range, it is the fractional free volume within the nanoparticle and its relationship to the free volume of the polymer that dictates the performance of these mixed matrix systems. Further, it shows that this performance can be readily predicted from the simple model presented by Equations 11 and 13, given the pore volume of the filler and the density of both polymer and filler are known. There is no need for more complex models of permeability. 
The data changes slightly when the $F F V$ term is modified to include the void volume (Equation (8)):

$$
\text { Total } F F V=F F V_{\text {polymer }}\left(\phi_{\text {polymer }}\right)+F F V_{\text {filler }}\left(\phi_{\text {filler }}\right)+\phi_{\text {voids }}
$$

In this case, there is some evidence that $\mathrm{Cu}-\mathrm{BTC}$ and ZIF-8 slightly outperform the more hydrophobic fillers. However, the correlation coefficient $\left(r^{2}\right)$ also decreases in this case, suggesting that a degree of error is being introduced. This probably reflects the dependence of void volume upon an accurate measurement of the nanoparticle density as discussed above. 


\section{CONCLUSION}

We have prepared defect-free self-standing MMMs from different nanoparticles embedded in a glassy polymer membrane, fabricated by the solvent casting method. Gas permeabilities of all MMMs were shown to increase with increasing filler loading without any reduction in the gas selectivity. Critically, the permeability of all gas species was shown to be strongly correlated with the free volume of the mixed matrix membrane and this factor was more relevant than the particle size or hydrophobicity. These results are likely to apply in all diffusivity dominated systems where the filler pore size is significantly larger than the penetrant size, as is the case with glassy polymers and many inorganic additives at low pressures.

\section{ACKNOWLEDGEMENT}

This research was supported by the Scientific and Industry Endowment Fund (SIEF), in Australia and Grant-in-Aid for the Japan Society for the Promotion of Science (JSPS) Fellows. 


\section{REFERENCES}

[1] D.R. Paul, D.R. Kemp, The diffusion time lag in polymer membranes containing adsorptive fillers, J. Polym. Sci.: Polym. Symp., 41 (1973) 79-93.

[2] H.J.C. te Hennepe, D. Bargeman, M.H.V. Mulder, C.A. Smolders, Zeolite-filled silicone rubber membranes: Part 1. Membrane preparation and pervaporation results, J. Membr. Sci., 35 (1987) 39-55.

[3] B.-H. Jeong, E.M.V. Hoek, Y. Yan, A. Subramani, X. Huang, G. Hurwitz, A.K. Ghosh, A. Jawor, Interfacial polymerization of thin film nanocomposites: A new concept for reverse osmosis membranes, J. Membr. Sci., 294 (2007) 1-7.

[4] M. Rostamizadeh, M. Rezakazemi, K. Shahidi, T. Mohammadi, Gas permeation through H2-selective mixed matrix membranes: Experimental and neural network modeling, Int. J. Hydrogen Energy, 38 (2013) 1128-1135.

[5] S. Kim, E. Marand, J. Ida, V.V. Guliants, Polysulfone and mesoporous molecular sieve MCM-48 mixed matrix membranes for gas separation, Chem. Mat., 18 (2006) 1149-1155.

[6] B.D. Reid, F.A. Ruiz-Trevino, I.H. Musselman, K.J. Balkus, J.P. Ferraris, Gas permeability properties of polysulfone membranes containing the mesoporous molecular sieve MCM-41, Chem. Mat., 13 (2001) 2366-2373.

[7] T.C. Merkel, B.D. Freeman, R.J. Spontak, Z. He, I. Pinnau, P. Meakin, A.J. Hill, Ultrapermeable, reverse-selective nanocomposite membranes, Science, 296 (2002) 519-522.

[8] D.Q. Vu, W.J. Koros, S.J. Miller, Mixed matrix membranes using carbon molecular sieves: I. Preparation and experimental results, J. Membr. Sci., 211 (2003) 311-334.

[9] A.F. Ismail, P.S. Goh, S.M. Sanip, M. Aziz, Transport and separation properties of carbon nanotube-mixed matrix membrane, Sep. Purif. Technol., 70 (2009) 12-26.

[10] M. Anson, J. Marchese, E. Garis, N. Ochoa, C. Pagliero, ABS copolymer-activated carbon mixed matrix membranes for CO2/CH4 separation, J. Membr. Sci., 243 (2004) 19-28.

[11] L. Ge, W. Zhou, V. Rudolph, Z. Zhu, Mixed matrix membranes incorporated with size-reduced Cu-BTC for improved gas separation, J. Mat. Chem. A, 1 (2013) 6350-6358.

[12] T.-H. Bae, J.S. Lee, W. Qiu, W.J. Koros, C.W. Jones, S. Nair, A high-performance gas-separation membrane containing submicrometer-sized metal-organic framework crystals, Angew. Chem. Int. Ed., 49 (2010) 9863-9866.

[13] Q. Song, S.K. Nataraj, M.V. Roussenova, J.C. Tan, D.J. Hughes, W. Li, P. Bourgoin, M.A. Alam, A.K. Cheetham, S.A. Al-Muhtaseb, E. Sivaniah, Zeolitic imidazolate framework (ZIF-8) based polymer nanocomposite membranes for gas separation, Energy Environ. Sci., 5 
(2012) 8359-8369.

[14] S. Japip, H. Wang, Y. Xiao, T. Shung Chung, Highly permeable zeolitic imidazolate framework (ZIF)-71 nano-particles enhanced polyimide membranes for gas separation, J. Membr. Sci., 467 (2014) 162-174.

[15] H. Zhao, Z. Jin, H. Su, J. Zhang, X. Yao, H. Zhao, G. Zhu, Target synthesis of a novel porous aromatic framework and its highly selective separation of $\mathrm{CO} 2 / \mathrm{CH} 4$, Chem. Comm., 49 (2013) 2780-2782.

[16] R. Mahajan, W.J. Koros, Mixed matrix membrane materials with glassy polymers. Part 1, Polym. Eng. Sci., 42 (2002) 1420-1431.

[17] M. Rezakazemi, A. Ebadi Amooghin, M.M. Montazer-Rahmati, A.F. Ismail, T. Matsuura, State-of-the-art membrane based CO2 separation using mixed matrix membranes (MMMs): An overview on current status and future directions, Prog. Polym. Sci., 39 (2014) 817-861.

[18] I. Erucar, S. Keskin, Computational screening of metal organic frameworks for mixed matrix membrane applications, J. Membr. Sci., 407-408 (2012) 221-230.

[19] J.D. Evans, D.M. Huang, M.R. Hill, C.J. Sumby, A.W. Thornton, C.J. Doonan, Feasibility of mixed matrix membrane gas separations employing porous organic cages, J. Phys. Chem. C, 118 (2013) 1523-1529.

[20] C. Maxwell, Treatise on Electricity arid Magnetism, Oxford University Press, London, 1873.

[21] H. Vinh-Thang, S. Kaliaguine, Predictive models for mixed-matrix membrane performance: A review, Chem. Rev., 113 (2013) 4980-5028.

[22] D.A.G. Bruggeman, The calculation of various physical constants of heterogeneous substances. I. The dielectric constants and conductivities of mixtures composed of isotropic substances, Ann. Phys. (Berlin, Ger.), 24 (1935) 636-664.

[23] T.B. Lewis, L.E. Nielsen, Dynamic mechanical properties of particulate-filled composites, J. Appl. Polym. Sci., 14 (1970) 1449-1471.

[24] B. Xiao, Q. Yuan, R.A. Williams, Exceptional function of nanoporous metal organic framework particles in emulsion stabilisation, Chem. Comm., 49 (2013) 8208-8210.

[25] C. Hua, A. Rawal, T.B. Faust, P.D. Southon, R. Babarao, J.M. Hook, D.M. D'Alessandro, Exploiting stable radical states for multifunctional properties in triarylamine-based porous organic polymers, J. Mat. Chem. A, 2 (2014) 12466-12474.

[26] G.Q. Chen, C.A. Scholes, C.M. Doherty, A.J. Hill, G.G. Qiao, S.E. Kentish, The thickness dependence of Matrimid films in water vapor permeation, Chem. Eng. J., 209 (2012) 301-312. 
[27] T.-S. Chung, S.S. Chan, R. Wang, Z. Lu, C. He, Characterization of permeability and sorption in Matrimid/C60 mixed matrix membranes, J. Membr. Sci., 211 (2003) 91-99.

[28] X. Duthie, S. Kentish, C. Powell, K. Nagai, G. Qiao, G. Stevens, Operating temperature effects on the plasticization of polyimide gas separation membranes, J. Membr. Sci., 294 (2007) 40-49.

[29] S.S.-Y. Chui, S.M.-F. Lo, J.P.H. Charmant, A.G. Orpen, I.D. Williams, A chemically functionalizable nanoporous material [Cu3(TMA)2(H2O)3]n, Science, 283 (1999) 1148-1150. [30] S. Basu, A. Cano-Odena, I.F.J. Vankelecom, Asymmetric Matrimid®/[Cu3(BTC)2] mixed-matrix membranes for gas separations, J. Membr. Sci., 362 (2010) 478-487.

[31] I. Senkovska, S. Kaskel, High pressure methane adsorption in the metal-organic frameworks Cu3(btc)2, Zn2(bdc)2dabco, and Cr3F(H2O)2O(bdc)3, Microporous Mesoporous Mater., 112 (2008) 108-115.

[32] T.C. Merkel, B.D. Freeman, R.J. Spontak, Z. He, I. Pinnau, P. Meakin, A.J. Hill, Sorption, transport, and structural evidence for enhanced free volume in poly(4-methyl-2-pentyne)/fumed silica nanocomposite membranes, Chem. Mater., 15 (2002) 109-123.

[33] Y. Zhang, K.J. Balkus Jr., I.H. Musselman, J.P. Ferraris, Mixed-matrix membranes composed of Matrimid® and mesoporous ZSM-5 nanoparticles, J. Membr. Sci., 325 (2008) 28-39.

[34] T.-S. Chung, L.Y. Jiang, Y. Li, S. Kulprathipanja, Mixed matrix membranes (MMMs) comprising organic polymers with dispersed inorganic fillers for gas separation, Prog. Polym. Sci., 32 (2007) 483-507.

[35] M.B. Rao, S. Sircar, Nanoporous carbon membrane for gas separation, Gas Separation \& Purification, 7 (1993) 279-284.

[36] Y.D. Chen, R.T. Yang, Concentration dependence of surface diffusion and zeolitic diffusion, AIChE Journal, 37 (1991) 1579-1582.

[37] H. Fujita, Diffusion in polymer-diluent systems, Fortschritte Hochpolymer- Forschung, 3 (1961) 1-47.

[38] J.Y. Park, D.R. Paul, Correlation and prediction of gas permeability in glassy polymer membrane materials via a modified free volume based group contribution method., J. Membr. Sci., 125 (1997) 23-39.

[39] S. Kanehashi, K. Nagai, Analysis of dual-mode model parameters for gas sorption in glassy polymers, J. Membr. Sci., 253 (2005) 117-138.

[40] A. Bondi, Physical Properties of Molecular Crystals, Liquids and Glasses, Wiley, 
NewYork, 1968.

[41] Y. Huang, X. Wang, D.R. Paul, Physical aging of thin glassy polymer films: Free volume interpretation, J. Membr. Sci., 277 (2006) 219-229. 


\section{Figure captions}

Figure 1 Particle size distribution of the Carbons (a) and MOFs (b), measured using dynamic light scattering.

Figure 2 Cross-sectional SEM images of pure Matrimid and $20 \mathrm{wt} \%$ MMMs

Figure 3 Relationship between void volume fraction of MMMs and (a) filler volume fraction and (b) water uptake at $0.21-0.22$ of filler volume fraction.

Figure 4 (a) $\mathrm{CO}_{2} / \mathrm{CH}_{4}$ and (b) $\mathrm{CO}_{2} / \mathrm{N}_{2}$ separation performance of MMMs using Matrimid polyimide as the host matrix. The dotted line represents the slope of the upper bound line at 2008, passing through the data point for pure Matrimid. The hollow circles represent a range of Matrimid-based MMM data from the literature.

Figure $5 \quad \mathrm{CO}_{2}$ permeability of MMMs as a ratio to the pure Matrimid permeability, as a function of the particle volume fraction at $35^{\circ} \mathrm{C}$ and 2 atm. Lines are based on the best fit to the data.

Figure $6 \quad \mathrm{CO}_{2}$ permeability enhancement ratio of MMMs at 0.21-0.22 of filler volume fraction at $35^{\circ} \mathrm{C}$ and $2 \mathrm{~atm}$ as a function of (a) void volume fraction, and (b) BET surface area of nanoparticle.

Figure 7 Relationship between the particle pore volume and the surface area as measured by BET sorption analysis.

Figure 8 (a) $\mathrm{CO}_{2}$ and $\mathrm{CH}_{4}$ and (b) $\mathrm{O}_{2}$ and $\mathrm{N}_{2}$ permeability of MMMs as a function of the inverse of the $F F V$ for $35^{\circ} \mathrm{C}$ and 2 atm. Lines are based on the best fit to Equation 13. 

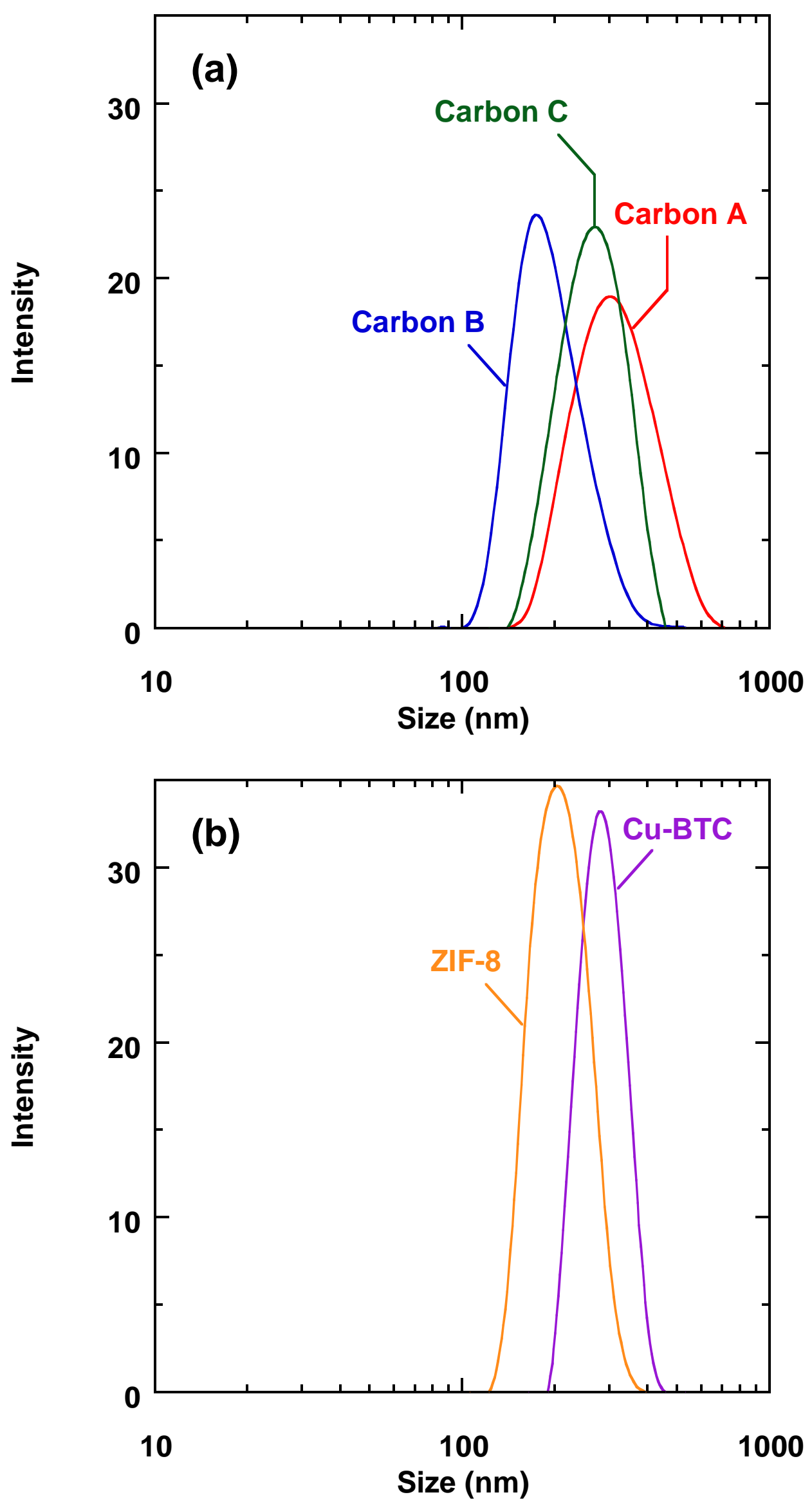

Figure 1 (Kanehashi et al.) 

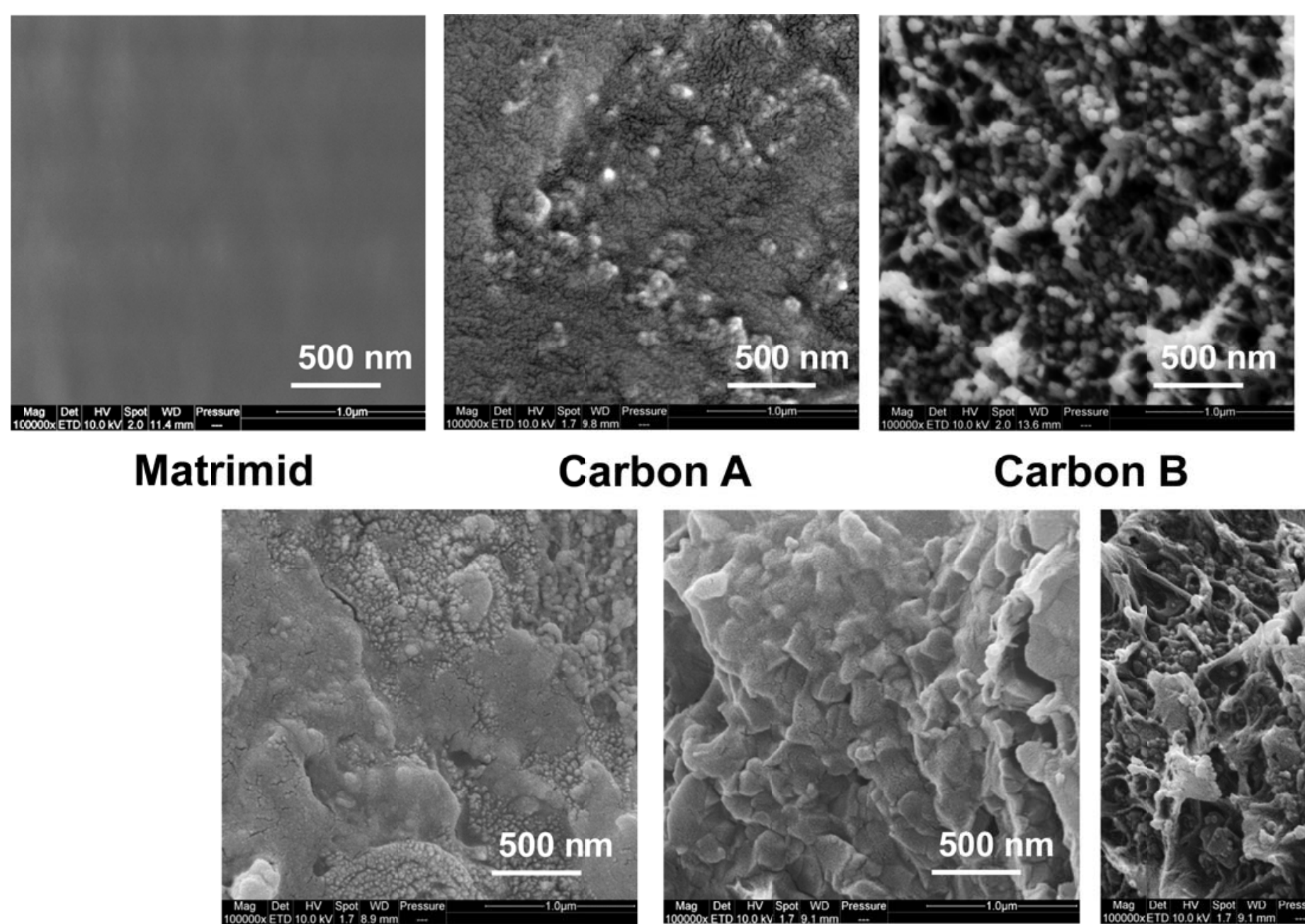

POP-2

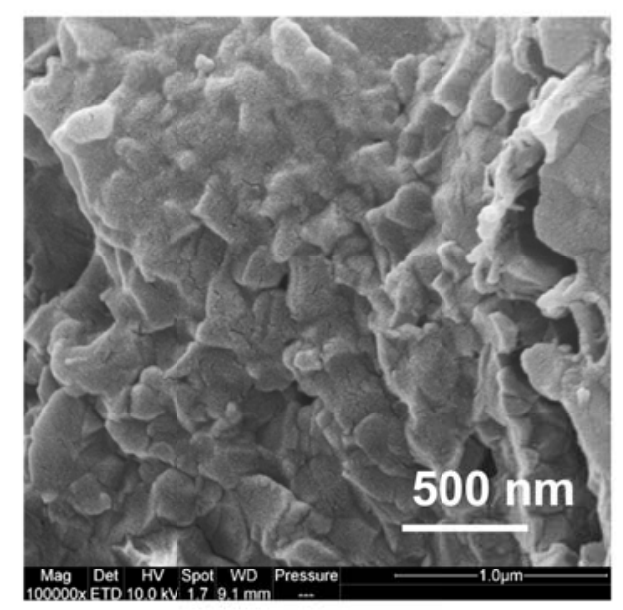

ZIF-8
Carbon B

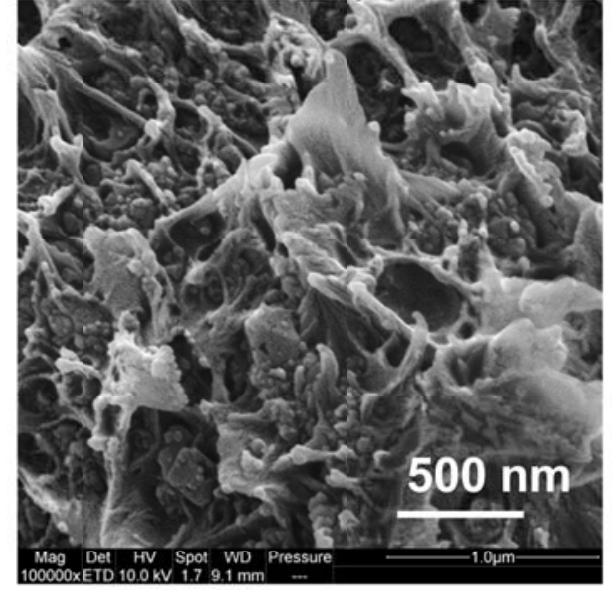

Cu-BTC

\section{Carbon C}

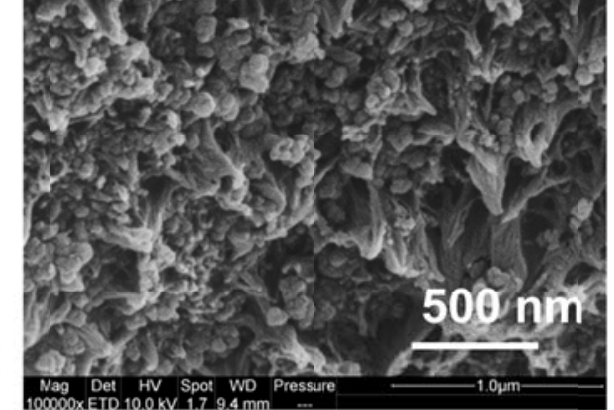




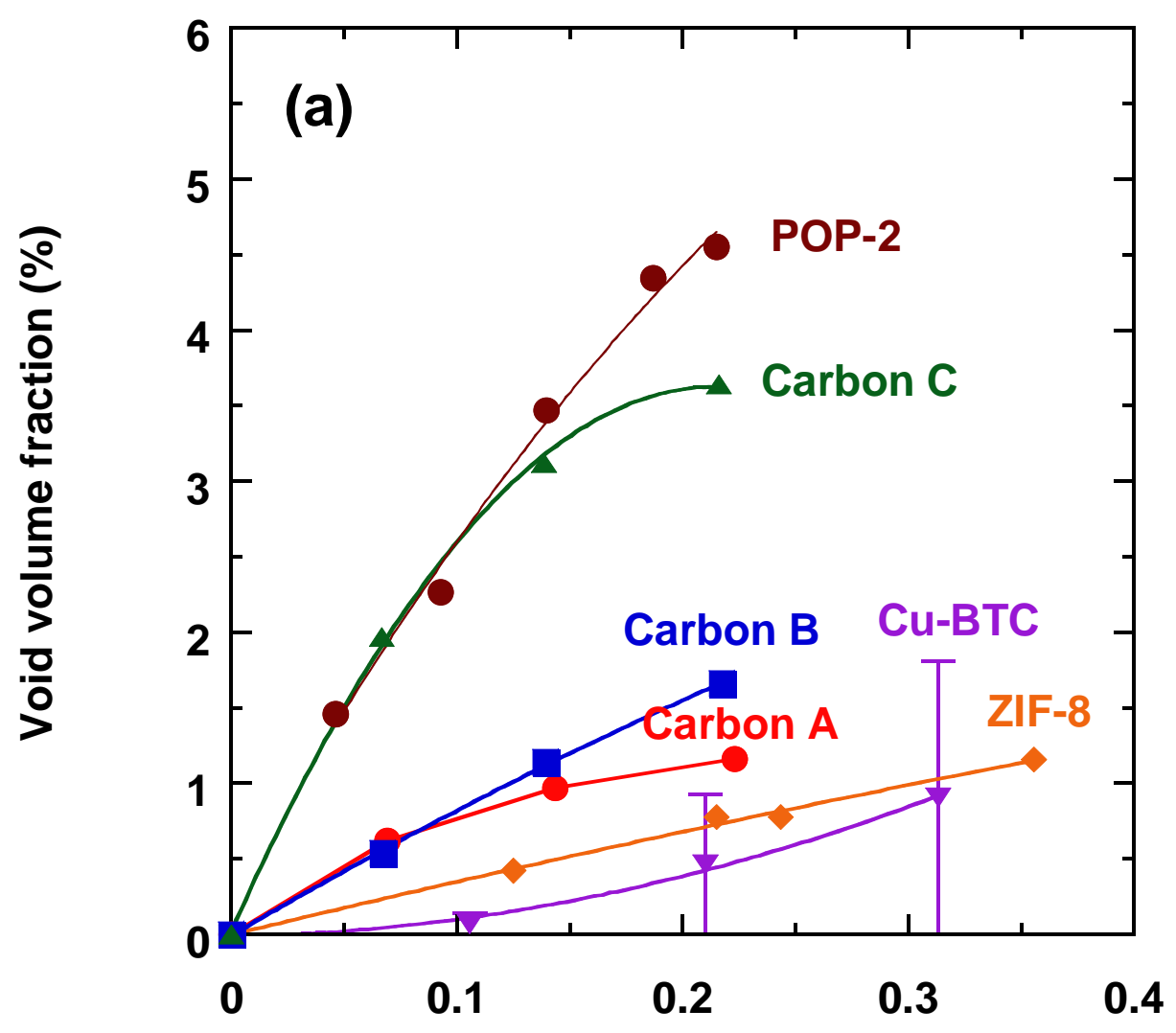

Filler volume fraction

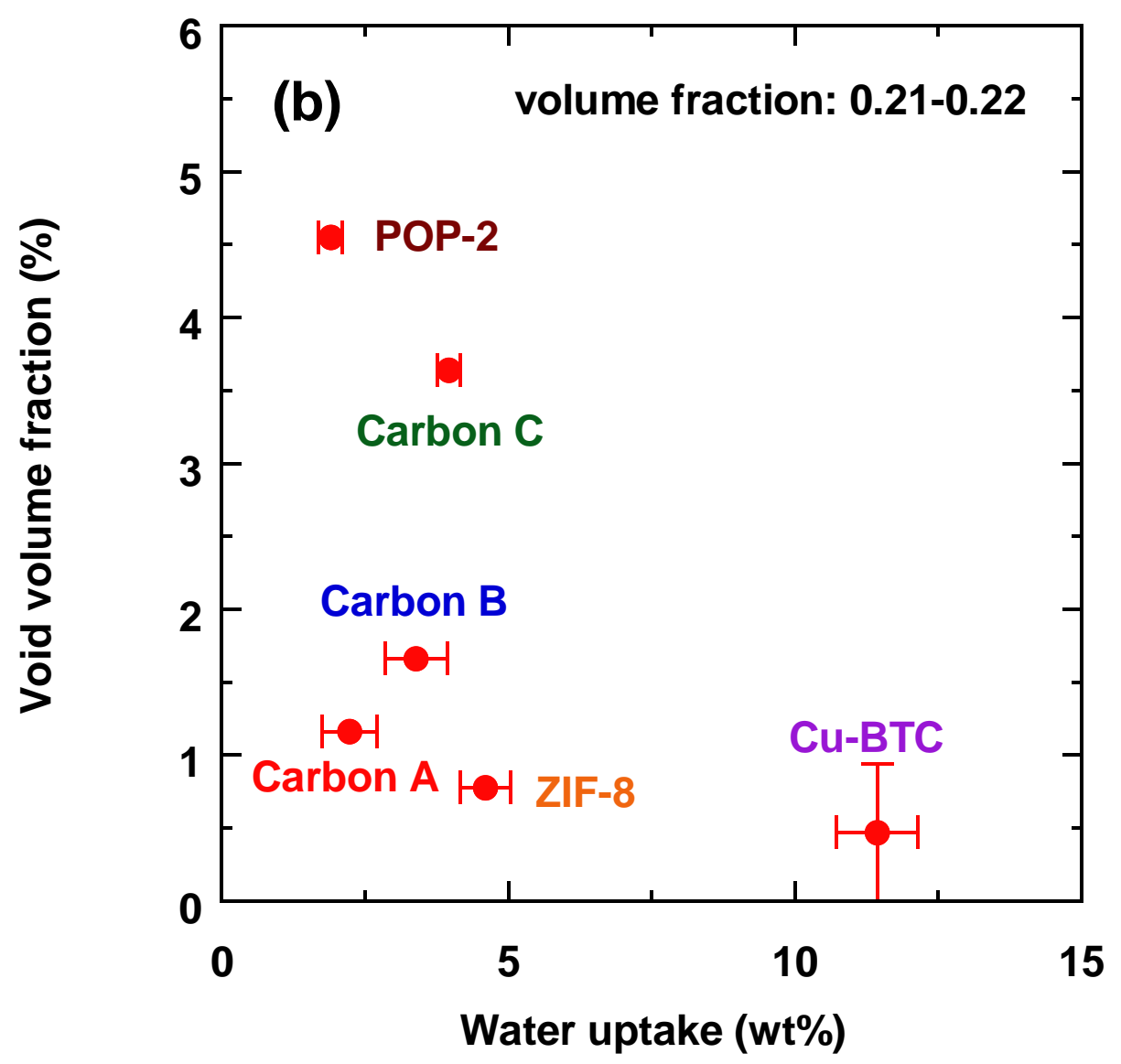



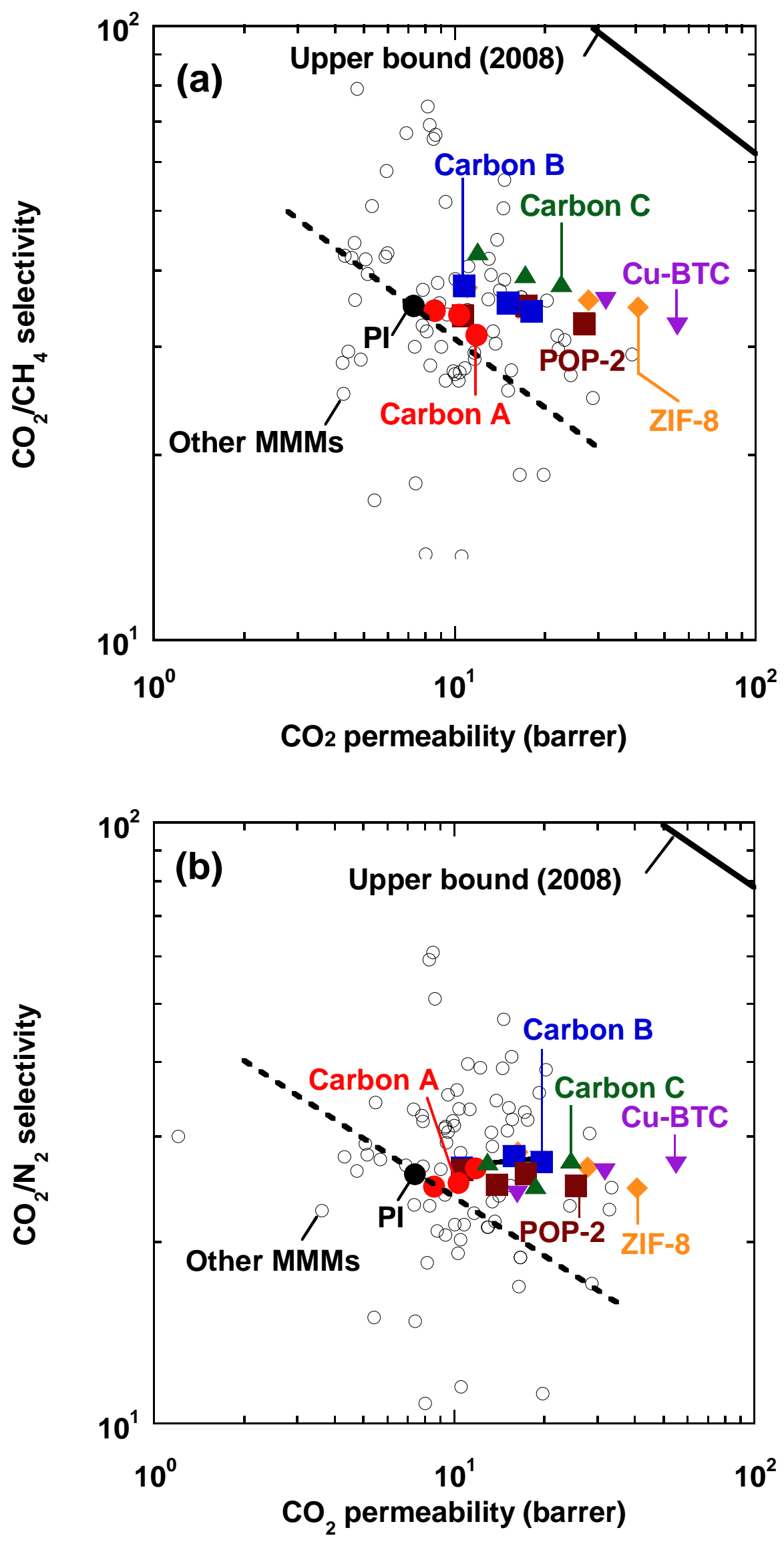


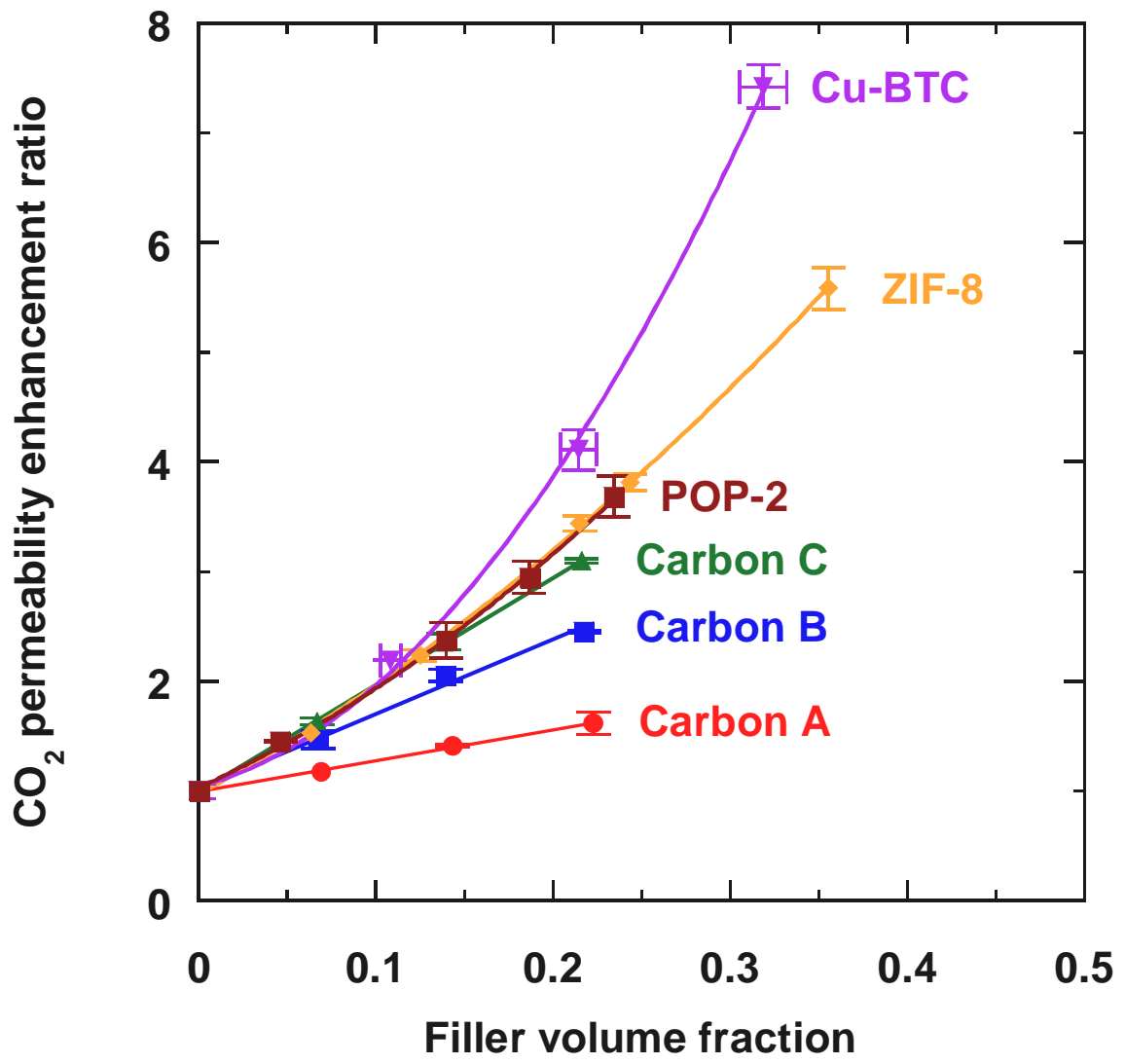




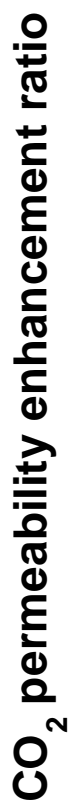

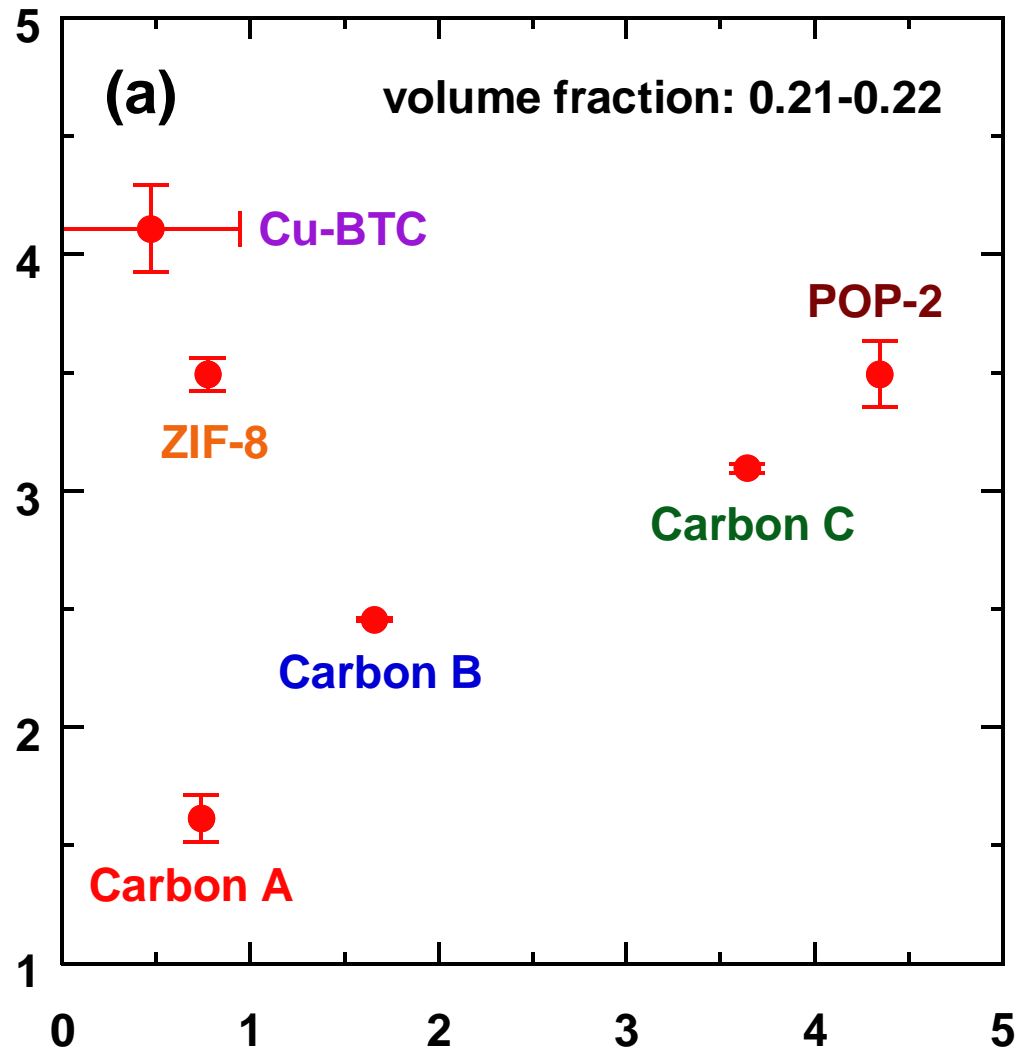

Void volume fraction (\%)

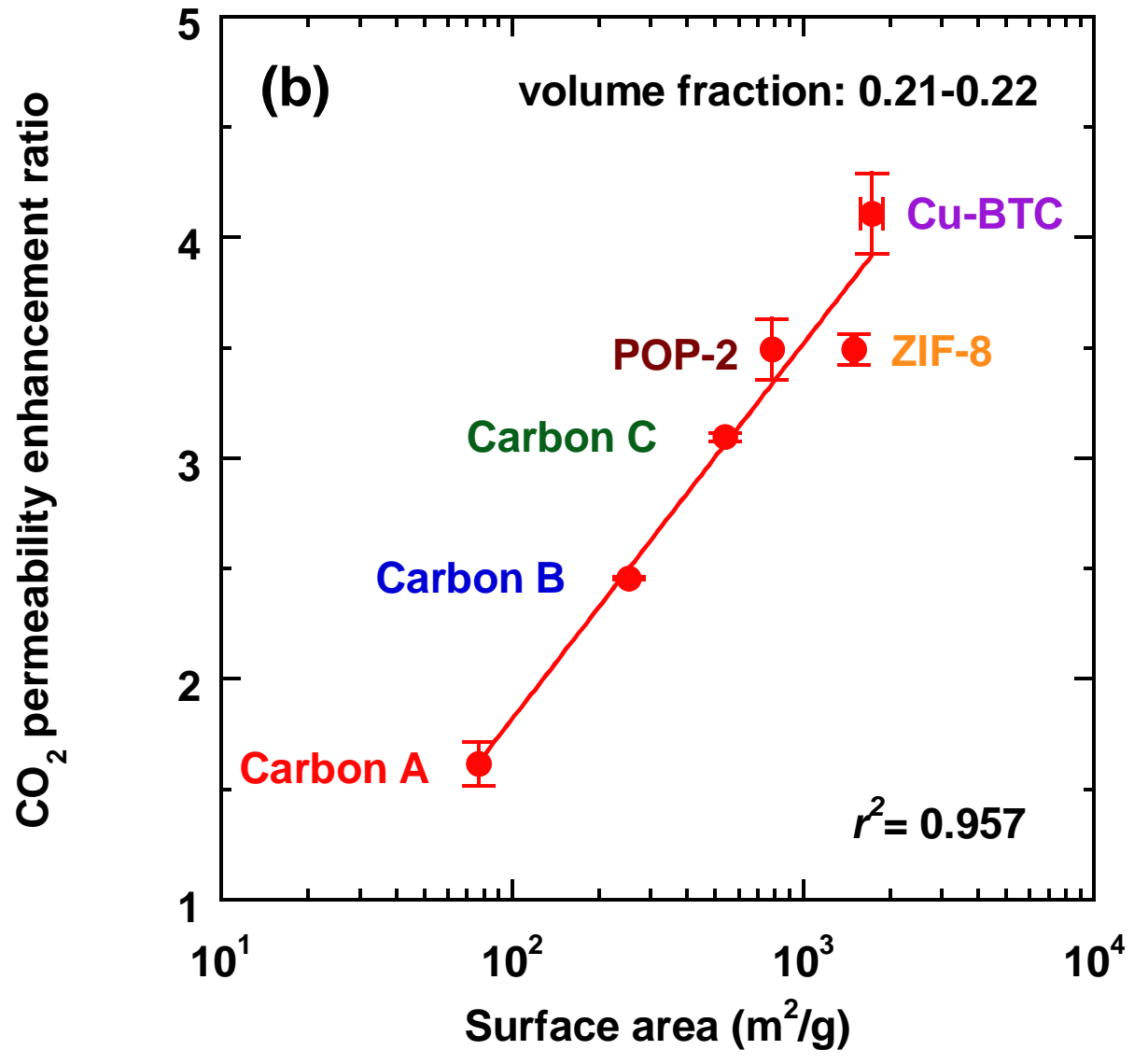




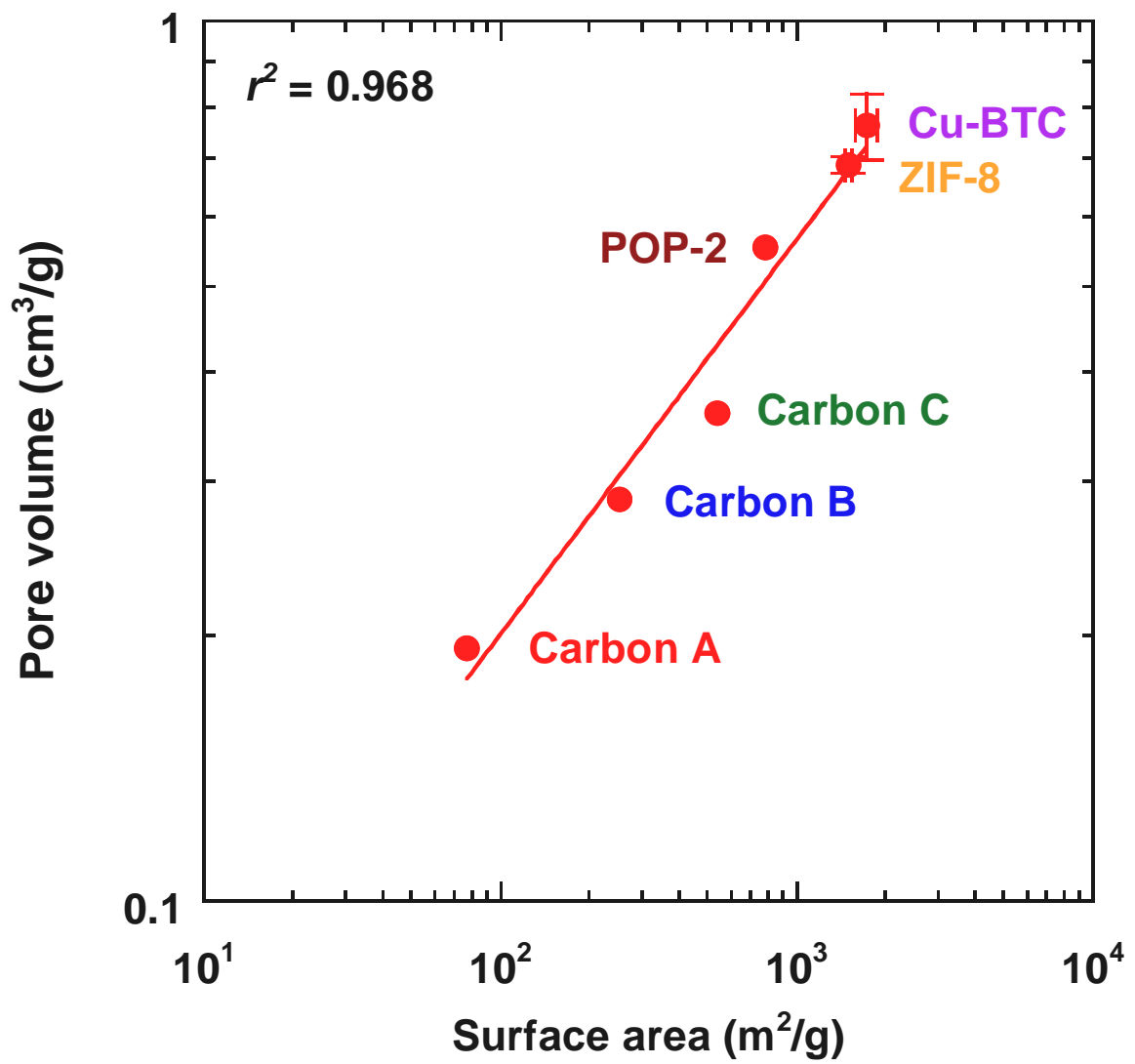



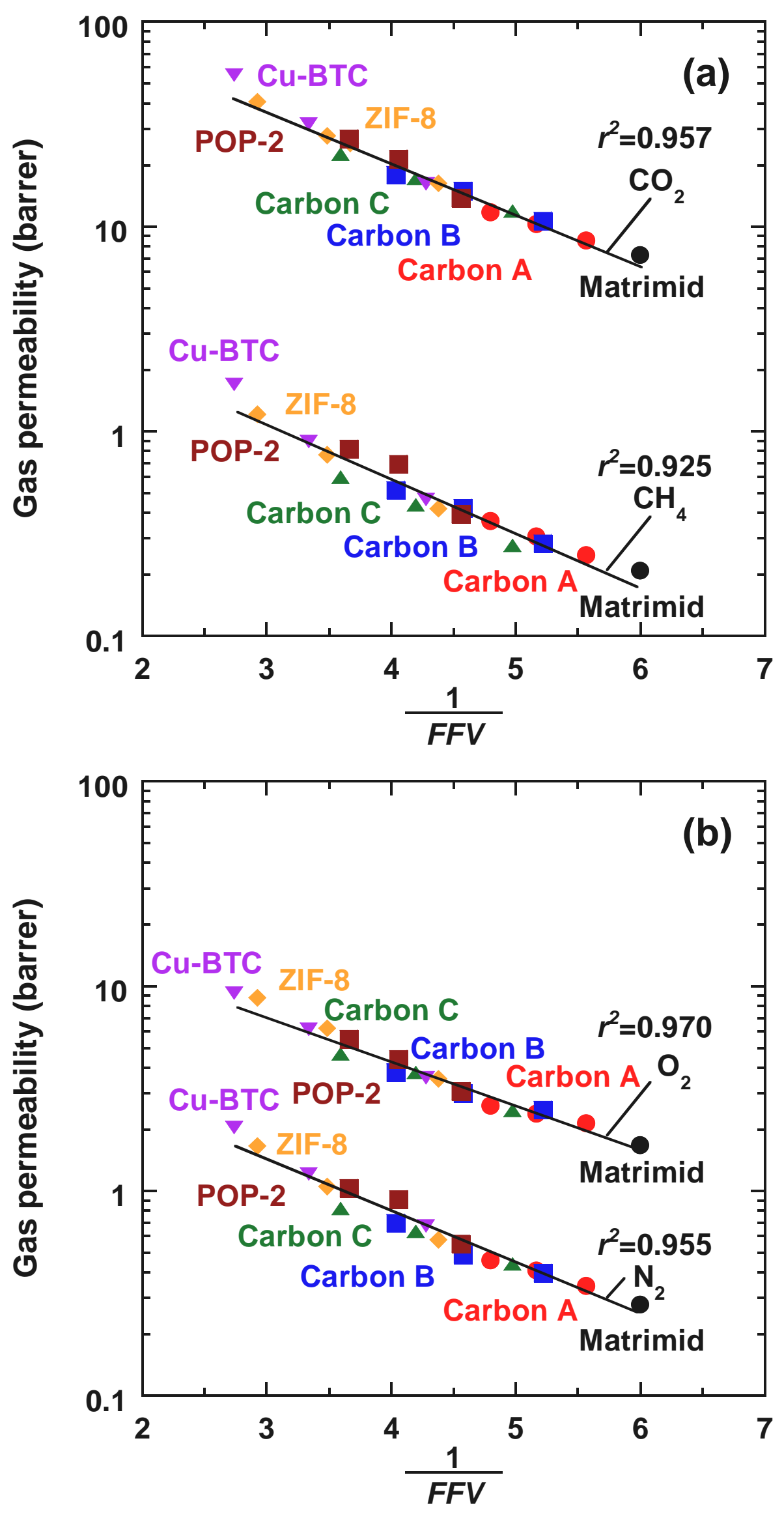
Table 1 Physical properties of the nanoparticles

\begin{tabular}{|c|c|c|c|c|c|c|}
\hline Property & Carbon A & Carbon B & Carbon $\mathrm{C}$ & POP-2 ${ }^{[25]}$ & ZIF-8 & $\mathrm{Cu}-\mathrm{BTC}$ \\
\hline Type & Carbon & Carbon & Carbon & POP & MOF & MOF \\
\hline Surface area $\left(\mathrm{m}^{2} / \mathrm{g}\right)$ & 77 & 252 & 540 & 781 & $1497 \pm 32$ & $1725 \pm 150$ \\
\hline Pore size $(\AA)$ & $137^{(b)}$ & $64^{(b)}$ & $20^{\text {(a) }}$ & $6,10,12.5$ & $3.4,11.6^{[13]}$ & $3.5,9.0^{[31]}$ \\
\hline Particle size $(\mathrm{nm})^{(\mathrm{c})}$ & 290 & 180 & 260 & $<300$ & 210 & 280 \\
\hline Water uptake $(\mathrm{wt} \%)^{(\mathrm{d})}$ & 2.2 & 3.4 & 4.0 & 1.9 & 5.0 & 7.8 \\
\hline
\end{tabular}

(a) Determined from $\mathrm{N}_{2}$ BET sorption analysis

(b) Cited from supplier information

(c) Average particle size of Carbons (A, B, and C) and MOFs (ZIF-8 and Cu-BTC) were measured by dynamic light scattering, while for POP-2 it was estimated from the SEM images

(d) For a $20 \mathrm{wt} \% \mathrm{MMM}$ at $35^{\circ} \mathrm{C}$ 